\title{
Tsafon
}

Revue d'études juives du Nord

$72 \mid 2016$

Juifs, Israéliens, dans la littérature française et israélienne

\section{Magdalena Kicińska, Pani Stefa}

Izabella Zatorska

\section{(2) OpenEdition}

Journals

Édition électronique

URL : https://journals.openedition.org/tsafon/496

DOI : $10.4000 /$ tsafon.496

ISSN : 2609-6420

Éditeur

Association Jean-Marie Delmaire

Édition imprimée

Date de publication : 1 décembre 2016

Pagination : 184-186

ISSN : 1149-6630

\section{Référence électronique}

Izabella Zatorska, « Magdalena Kicińska, Pani Stefa », Tsafon [En ligne], 72 | 2016, mis en ligne le 31 mai 2018, consulté le 24 juin 2021. URL : http://journals.openedition.org/tsafon/496 ; DOI : https:// doi.org/10.4000/tsafon.496

Ce document a été généré automatiquement le 24 juin 2021.

Tsafon. Revues d'études juives du Nord 


\title{
Magdalena Kicińska, Pani Stefa
}

\author{
Izabella Zatorska
}

\section{RÉFÉRENCE}

Magdalena Kicińska, Pani Stefa, Wołowiec, Wydawnictwo Czarne, coll. „Reportaż”, 2015, 266 p.+6 nlb, 16 photographies

Difficile d'écrire une histoire dont la fin est connue depuis longtemps. On peut alors chercher à en éclaircir les motifs et les conséquences, ou les tenants et les aboutissants, les faits mêmes s'estompent. Cependant, pour bien apprécier ce qui contribue aux événements et ce qui en résulte, la pénétration des conditions matérielles et spirituelles elles-mêmes est inappréciable. D'autant si la clé de l'énigme repose dans l'âme ou le caractère des personnes en question. Et voilà ce que tend à restituer, avec des moyens modernes, variés et contrastés, Magdalena Kicińska dans sa biographie de Stefania Wilczyńska (1886-1942), récit documenté plus que romancé.

"Madame Stefa », ainsi les enfants de l'orphelinat du 92 rue Krochmalna ont appelé l'assistante du docteur Janusz Korczak (nom de plume popularisé; son vrai nom de famille est Henryk Goldszmidt). La société «Assistance aux Orphelins» (« Pomoc dla sierot ») avait géré les donations (depuis 1910 les premières affluent) qui ont permis de construire la "Maison des Orphelins » en question, puis une école maternelle, une bourse pour les anciens élèves souhaitant poursuivre leurs études au-delà de 14 ans, l'âge limite (sauf exception) pour vivre à l'abri de la République d'Enfants. Le président de cette société (fondation), le Dr Isaac Eliasberg, fut le beau-père de Stanislas Wilczynski, frère de Stefania ; ce dernier et sa femme Irena ont survécu loin du ghetto, n'ayant jamais révélé leur ascendance juive. Lui mourra d'un cancer (dit-on) en 1943 et sera inhumé au cimetière catholique de PowĄzki. Chose incroyable, personne n'aura l'idée d'interroger sa veuve dont l'une des sœurs, Helena épouse Szymon Syrkus, fut célèbre comme architecte, avec son mari, de " quartiers sociaux », beaux quartiers pour les ouvriers et l'intelligentsia installés dans une fraternité utopique. On peut toujours visiter leurs vestiges sur la rive droite de Varsovie, à Grochów. Personne n’a cherché à 
interroger le célèbre prêtre poète Jan Twardowski, mort en 2006, sur ses relations avec les sœurs Eliasberg : lui-même les aurait préparées au baptême catholique et baptisées (sous l'occupation?); après la Seconde Guerre, Irena, ancienne combattante de l'Insurrection de Varsovie en 1944, sera membre du parti communiste et catholique pratiquante. Le P. Jan aurait aussi connu Stefania dont il a parlé avec des superlatifs, une notice aux archives de Korczak en témoigne.

Les effectifs de l'établissement, inauguré en 1912, vont de 150 à plus de 200 enfants (dans la prospérité comme dans le malheur le chiffre a tendance à croître). D'entrée de jeu son personnel - et notamment la responsable en chef, Stefania, née en 1886 et forte de ses études en chimie et en botanique à Genève (semestre d'été 1906) et à Liège ("sciences naturelles et médicales», 1906/1907, 1907/1908) - sera confronté aux pénuries de la Grande Guerre ; la crise économique de 1929-1934 se poursuivra, cette fois sur le fond spirituel: le découragement gagne les deux responsables qui, tour à tour, entraînés par leurs anciens élèves séduits par le rêve sioniste, font des essais d'adaptation en Palestine : Stefania quitte le kibbutz Ein Charod, où sa disciple et amie Fejga Lifszyc avait fini par fonder une famille, à la mi-mai 1939 pour revenir fêter son $53^{\text {ème }}$ anniversaire, à Varsovie le 26 mai.

Le 5 ou le 6 août 1942, avec le docteur Korczak, une centaine d'orphelins, répartis en deux wagons, ont quitté Umschlagplatz pour Treblinka. Peu de documents sont restés dans le dossier que l'auteure, Magdalena Kicińska, a réussi à établir, principalement en fouillant le Korczakianum ou les archives de Janusz Korczak, déposées dans le bâtiment de l'orphelinat qui lui a survécu; la rue seulement a changé de nom : de Krochmalna elle est devenue Jaktorowska. La maison porte le nom de Janusz Korczak. Bien que souvent remaniée, celle-ci aurait gardé le carrelage original dans le couloir de la cuisine (voir la photo de «La Maison des Enfants " $\mathrm{n}^{\circ} 2$, dédiée à Janusz Korczak: 6 rue Jaktorowska, p. 167).

5 Née dans une famille peu à peu " assimilée » depuis trois générations (son grand-père paternel avait été marchand à Nieszawa, son père se nommait tantôt Isaac, tantôt "[vel] Julian », les actes de naissance de ses enfants et son acte de décès le définissent comme « commerçant » et/ou comme banquier : un dédoublement possible à cause du changement de prénom?), Stefania ne parle chez elle que polonais, apparemment, puisqu'elle doit apprendre le yiddish pour communiquer avec les orphelins ou les enfants des milieux défavorisées pris aussi en charge par la "Maison des Orphelins " (nom officiel de l'établissement); et l'hébreu lorsqu'elle se prépare à ses départs successifs en Palestine : elle se plaint de ses progrès très lents, elle n'a pas de temps et puis son âge dispose peu à apprendre des langues alors qu'elle avait dû en acquérir au moins trois dans sa jeunesse (le russe, le français, l'anglais et peut-être l'allemand).

6 Elle a mené deux trajets parallèles et elle a fini par choisir celui qui l'a fait demeurer en Pologne, sans croire dans le triomphe de ses idées dans un monde hostile, qui remettra en cause jusqu'à l'utilité de la formation obtenue par les enfants, celle qui s'est avérée tragique finalement.

7 L'un des anciens pupilles - parmi les rares survivants, retrouvés et interrogés par la journaliste, plus rares encore sont ceux qui ont gardé des bribes de souvenirs - cité par l'auteure, conclut ainsi le $16^{\mathrm{e}}$ et avant-dernier chapitre sur la décision finale de Wilczyńska et de Korczak: "Si lui ainsi que Stefa avait passé toute leur vie de cette sorte, et pas d'une autre, avec nous, comment auraient-ils pu choisir autrement ? ! Là- 
bas, il n'y a eu aucun choix ! Aucun choix, comprends-tu ?! Juste une possibilité toute théorique » (p. 246).

Le même Szlomo Najdel, à qui Magda Kicińska rend visite à Ramla, s'empresse de l'assurer, en ouverture du récit : « Il existe un paradis, dit-on. [...] Un paradis ! J'ignore où ils puisent leur certitude. C'est moi qui puis en être sûr. Car j'y ai déjà été. Personne n'est jamais revenu du paradis, pour raconter. Sauf moi. Car j'ai bien été dans une utopie. J'ai vécu heureux. On m'a enseigné comment faire pour l'être. J'avais eu une maison, où le papa c'était Stefa, et la maman, Korczak » (p. 9). Le caractère ferme, la rigueur (appliquée d'abord à soi-même) distinguent "Madame Stefa » au dire de ses anciens. Elle a noué pourtant quelques amitiés, en tant que responsable de la Maison (on ignore tout de sa jeunesse et de ses contacts à l'étranger): Esthère Weintraub, éduquée en Suisse aux frais de Stefa, stagiaire et aide à l'orphelinat, fut sa première amie connue ; elle mourut de la fièvre typhoïde contractée auprès d'enfants malades au début de 1918. Depuis, c'est surtout avec Fejga Lifszyc qu'elle maintiendra un contact, la conseillera dans la relation avec son futur mari, sera une «mamie» pour son fils, mais sans se résoudre à la suivre définitivement en Palestine.

Magdalena Kicińska, jeune journaliste mais expérimentée, sait guider son lecteur à travers des sources sans l'ennuyer: les informations quantitatives sont simplement citées (distribution de provisions au temps des guerres et des crises, bilan difficile de fonds précaires, maladies d'enfants) ou commentées d'une manière sobre, laissant au lecteur la liberté d'en tirer ses propres conclusions. La presse n'est pas très présente, contrairement aux témoignages d'anciens pupilles eux-mêmes: les détails sur la vie vers la fin de la Grande Guerre sont fournis avec une simplicité poignante par le journal d'une anonyme « fille $n^{\circ} 56$ " (les enfants ont chacun leur numéro, mais cela n'autorise aucune prémonition tragique: l'auteure nous rassure qu'il s'agissait de ne pas confondre le linge et les vêtements au lavage, les maladies chroniques de peau notamment rendaient cette précaution prioritaire); beaucoup de renseignements ont été préservés grâce aux interviews recueillis par Ada Poznanska-Hagari, une sociologue d'Israël, vers 1980. Car, à défaut de pièces d'archives, l'ultime dépôt devient de nouveau, comme dans les siècles révolus, la mémoire des particuliers. Heureusement, l'auteure fournit une bibliographie sélective (quatre pages) à la fin de son volume; la liste des vivants qu'elle remercie (tout une page) est aussi très longue. Mais nous, ses lecteurs, devrons d'abord remercier Magdalena Kicińska pour son effort de comprendre. 